\title{
DOSSIER:
}

\section{Osteometría de camélidos sudamericanos}

RMA

Dossier - Arqueología
Andrés D. Izeta

CONICET, Museo de Antropología, Facultad de Filosofía y Humanidades, Universidad Nacional de Córdoba. E-mail: andresizeta@gmail.com

\begin{abstract}
La identificación de elementos óseos y su asignación a una especie animal concreta es un tema central para la zooarqueología. Por ello desde la década de 1970 se han estudiado las diferencias morfométricas que permiten la diferenciación de especies que comparten una morfología ósea similar, especialmente cuando ésta es el resultado de un proceso de domesticación o de mejora de alguno de los caracteres de la población (e.g. Albarella y Payne 2005, von den Driesch 1976, Davis 1996, Payne y Bull 1988, Reitz 1994, Uerpmann 1979, Weinstock 2003 y bibliografía allí citada). Esta similitud morfológica puede tener sus dificultades en casos particulares como el de los camélidos sudamericanos. Esta familia (Camelidae) está compuesta actualmente por cuatro especies: Lama guanicoe Muller 1776 (guanaco), Lama glama Linnaeus 1758 (llama), Lama pacos o Vicugna pacos Linnaeus 1758 (alpaca) y Vicugna vicugna o Lama (Vicugna) vicugna Molina 1782 (vicuña).
\end{abstract}

Ante la presencia de tantas variedades diferentes de camélidos es sumamente importante el estudio de las variaciones morfológicas, que permitan diferenciar las distintas especies, tema al que la osteometría pretende aportar nuevos datos para esta familia tan conspicua en el registro arqueológico y paleontológico sudamericano. Esto es una cuestión clave si lo que se intenta es realizar una reconstrucción paleoeconómica de las poblaciones humanas del pasado o de la paleoecología del ambiente en donde desarrollaron sus actividades.

En los últimos años se ha implementado la aplicación de distintas técnicas sobre los restos de camélidos con el fin de avanzar en el análisis de la composición de los conjuntos recuperados de sitios arqueológicos de varias regiones del país y de países limítrofes (Noroeste argentino, Patagonia argentina y norte de Chile).

Estas variaciones han sido estudiadas a través de dos vías de análisis: uno de tipo cualitativo y otro cuantitativo. Las formas de diferenciación entre especies basadas en análisis de tipo cualitativo corresponden al estudio de la morfología dental de los incisivos (Wheeler 1982, Lavallée et al. 1995: 58-59) y el estudio de las fibras (lanilla y pelo) (Reigadas 1994, 2001). El otro tipo de técnica que permite la identificación de las distintas especies corresponde a los análisis cuantitativos a partir de la osteometría y la aplicación de análisis estadísticos sobre sus resultados. Una de las primeras aplicaciones de esta clase de estudios sobre camélidos de contextos arqueológicos fue realizada por Jonathan Kent (1982). Otros autores lo han seguido como Elkin (1996), Izeta (2004, 2006 e. p.), López (2003), Madero (1992, 1993-1994) y Yacobaccio et al. (1997-1998) para el Noroeste de la Argentina y L'Hereux (2005) para Patagonia. En el ámbito de la Paleontología también se han realizado diversos estudios aplicando este tipo de análisis como los de Menegaz $(2000)$ y Menegaz et al. $(1988,1989)$ entre otros.

Por ello este Dossier tiene como fin agrupar diversos trabajos que enfocan en esa diversidad dentro de la familia Camelidae con el fin de logar diversas interpretaciones. Estas van desde cuestiones relacionadas con la identificación de caracteres métricos que permitan definir sexo (Kaufmann y L'Heureux), variaciones métricas entre especies domésticas (Vásquez y Rosales), entre especies domésticas y silvestres (Cartajena, Izeta et al.), entre especies silvestres (Labarca y Prieto) y patrones económicos (Olivera y Grant).

Por otro lado es notable que el análisis e interpretaciones sustentadas en datos osteométricos se basen en muy pocos ejemplares comparativos razón por la cual todos los autores concuerdan en que puede existir algún grado de error que no nos permita interpretar adecuadamente los resultados que se están obteniendo. Esto es especialmente importante para el área norandina donde la diferenciación entre especies de camélidos se basa en los valores osteométricos conseguidos de las mediciones de tres ejemplares (dos guanacos norandinos y una llama -ver Elkin 1996 e Izeta 2004) y las mismas se han centrado en su mayoría en los datos de las falanges proximales. Por ello este Dossier 
también adquiere relevancia ya que todos los trabajos que aquí se presentan poseen los datos sobre los cuales se efectúan las interpretaciones, poniendo por primera vez y a disposición de los colegas una base de datos amplia que incluye valores métricos de todas las especies de camélidos que se pueden hallar en contextos arqueológicos y aun paleontológicos de Sudamérica, cubriendo un espectro regional y temporal amplio.

Otro aspecto a destacar es que aquí se brindan seis aportes que utilizan diversas técnicas analíticas a partir de los valores osteométricos. En el caso de Vásquez y Rosales se aplica un análisis discriminante a partir de scores calculados en conjuntos de camélidos modernos de la Puna peruana. Este trabajo sigue la vía iniciada por Jonathan Kent hace más de 25 años atrás, la cual no ha sido muy utilizada en nuestro medio. No obstante ello los autores logran mediante esta técnica interpretar conjuntos que poseen individuos producto de la cruza de distintas especies (huarizos). El trabajo de Labarca presenta datos novedosos acerca de la presencia de un camélido pequeño para el sur de Patagonia que identifica como vicuña. Esta interpretación, basada en agrupamientos de conglomerado y Análisis de Correspondencias, confirma el resultado obtenido previamente mediante análisis moleculares (Weinstock et al. 2009). Olivera y Grant comparan ocupaciones en puestos de altura y los restos de camélidos asignándolos a diversas especies mediante análisis univariados como el Logarithmic Size Index (LSI) de Meadow (1989) y análisis de conglomerados. Cartajena aplica varios análisis con el fin de determinar diferencias en tamaño entre los camélidos pequeños (vicuña y alpaca), siendo este uno de los primeros trabajos en encarar esta problemática. Kaufmann y L'Heureux utilizan análisis univariados (ANOVA) y multivariados con el fin de determinar variables que puedan ser utilizadas para diferenciar sexo en una especie etiquetada como sexualmente no dimórfica. Por último Izeta, Otaola y Gasco presentan una combinación de diversos estadísticos y representaciones gráficas utilizadas comúnmente cuando se intenta discriminar entre especies con el fin de alertar mediante casos concretos la dificultad que conlleva realizar interpretaciones a partir de conjuntos comparativos modernos cuando no se tiene en cuenta la variabilidad intraespecífica dada por diversos motivos como variaciones geográficas (variaciones latitudinales).

Por supuesto que con este dossier no se agota la temática pero es un esfuerzo colectivo que incluye a los autores y a los evaluadores de cada trabajo y que tan solo pretende ofrecer un estado del arte de este tipo de aproximación en el cual puede observarse una gran actividad de investigadores en todo el continente.

Córdoba, 14 de Noviembre de 2009 